\title{
Respuestas foliares de Aristotelia chilensis (Molina) Stuntz (Elaeocarpaceae) a la fragmentación del bosque maulino
}

\author{
Leaf responses of Aristotelia chilensis (Molina) Stuntz (Elaeocarpaceae) to the \\ fragmentation of the Maulino forest
}

\author{
FIORELLA REPETTO-GIAVELLI ${ }^{1}$, LOHENGRIN A. CAVIERES $^{2,3}$ \\ \& JAVIER A. SIMONETTI ${ }^{1, *}$
}

\footnotetext{
${ }^{1}$ Departamento de Ciencias Ecológicas, Facultad de Ciencias, Universidad de Chile. Casilla 653, Santiago, Chile

${ }^{2}$ Departamento de Botánica, Facultad de Ciencias Naturales y Oceanográficas, Universidad de Concepción, Casilla 160C, Concepción, Chile

${ }^{3}$ Instituto de Ecología y Biodiversidad, Santiago, Chile

*e-mail para correspondencia: jsimonet@uchile.cl
}

\begin{abstract}
RESUMEN
La fragmentación que ha sufrido el bosque nativo de Chile debido a la fuerte presión antrópica ha causado, además de la grave pérdida de hábitat, la modificación del microclima de los parches de bosque remanente que alguna vez constituyeron un bosque continuo de especies nativas. Estos cambios generarían respuestas morfológicas, químicas y fisiológicas en plantas capaces de adaptarse a las nuevas condiciones. Este estudio tiene como objetivo identificar respuestas a nivel de las hojas ante el aumento de radiación solar y disminución de agua en el suelo que ocurre al interior de los fragmentos. Para esto utilizamos a Aristotelia chilensis, especie que crece tanto en fragmentos como en bosque continuo, y comparamos parámetros relacionados a su morfología foliar en bosque y fragmentos y medimos su repercusión en la capacidad fotosintética de A. chilensis. En términos morfológicos, se observó una disminución del área foliar y del área foliar específica en los fragmentos, siendo 1,2 veces menor que en el bosque continuo. En los fragmentos, el grosor de la epidermis y del parénquima esponjoso son más de 1,3 veces mas gruesos que en el bosque continuo. El grosor del parénquima en empalizada, en cambio, no se vio modificado. La cantidad de nitrógeno en las hojas es 1,2 veces mayor en el bosque continuo que en los fragmentos, mientras que el contenido de carbono no varía. La conductancia estomática en el bosque continuo fue 1,5 veces mayor que en los fragmentos. Aristotelia chilensis responde morfológica y fisiológicamente ante los cambios abióticos generados por la fragmentación de los bosques, lo que le permite sobrevivir tanto en ambientes de baja luminosidad como el bosque continuo y en ambientes de alta luminosidad y bajo contenido hídrico como los fragmentos de bosque, manteniendo tasas fotosintéticas semejantes en ambos ambientes.
\end{abstract}

Palabras clave: Chile, ecofisiología, fragmentación, morfología foliar.

\begin{abstract}
Fragmentation of the Maulino forest implies significant habitat loss, as well as the modification of the microclimatic conditions of the remaining forest patches. Fragments are drier, hotter and receive more light than the continuous forest. These changes might induce morphological, chemical and physiological responses on individuals inhabiting forest patches. This study aims to identify morpho and physiological changes in Aristotelia chilensis, an evergreen tree that grows both in forest fragments and continuous forest. Leaves were 1.2 times smaller in forest fragments than in the continuous forest. Similar reduction was observed on specific leaf area (SLA). In forest fragments, the thickness of epidermis and of the spongy mesophyll was more than 1.3 times larger than that on the continuous forest, whereas the thickness of the palisade mesophyll did not differ. The amount of foliar nitrogen was 1.2 times larger in the continuous forest than in fragments, whereas the carbon content did not differ. Stomatal conductance in the continuous forest was 1.5 fold higher than that in forest fragments. Changes depicted by A. chilensis allows it to survive in environments of low luminosity as the continuous forest, and in environments of low water content as the forest fragments, maintaining similar photosynthetic rates in both environments.
\end{abstract}

Key words: Chile, ecophysiology, fragmentation, foliar morphology. 


\section{INTRODUCCIÓN}

La fragmentación de los bosques modifica el hábitat remanente tanto a nivel biótico como abiótico (Murcia 1995). Generalmente, la intensidad lumínica y la temperatura del aire son mayores en fragmentos de bosque que en un bosque continuo, mientras que la humedad del suelo y del aire son menores, generando una diferencia de presión de vapor (DPV) entre las hojas y el aire mayor en los fragmentos que en el bosque continuo (Kapos et al. 1997). La mayor DPV implica mayores demandas de evapotranspiración para las plantas. Al variar tanto la radiación solar como el agua disponible para las plantas, procesos como la fotosíntesis y transpiración foliar pueden ser afectados. Frente a estos cambios ambientales las plantas podrían combinar respuestas de tipo demográficas, morfológicas, bioquímicas y fisiológicas, respondiendo en forma análoga a como lo harían frente a variaciones ambientales que ocurren naturalmente en distintos hábitats (e.g., Harper 1980, Beerling \& Chaloner 1993, Davies \& Cowing 1999, Pearcy 1999).

Dentro de las respuestas morfológicas, bioquímicas y fisiológicas de las plantas frente a variaciones en su entorno abiótico, hojas de una misma especie desarrolladas en condiciones de alta luminosidad (como las que se podrían encontrar en fragmentos de bosque) tienden a ser más gruesas, con parénquima en empalizada de mayor grosor y mayor cantidad de capas de parénquima esponjoso que hojas desarrolladas bajo sombra (Givnish 1988, Chazdon \& Kaufmann 1993, Silva et al. 2001). Hojas desarrolladas en alta luminosidad también poseen menor cantidad de nitrógeno por masa seca y una mayor relación $\mathrm{C} / \mathrm{N}$ por acumulación de carbono, lo que resulta en una mayor síntesis de compuestos de defensa (Leal \& Thomas 2003). Por otro lado, respuestas foliares como disminución en la densidad estomática, cierre de estomas, menor área estomática y mayor grosor de la epidermis y del tejido fotosintético confieren resistencia a las hojas contra la pérdida de agua en situaciones de sequía (Schulze \& Hall 1982, Buckley 2005).

Por el contrario, plantas expuestas a baja luminosidad, como ocurre en un bosque continuo, presentan mayor área foliar específica (SLA) que aquellas expuestas a alta luminosidad (Givnish 1988, Lusk \& Del Pozo 2002), y la producción de compuestos basados en el nitrógeno en las hojas es mayor (Lerdau 2002). Ambas modificaciones incrementarían la eficiencia fotosintética a baja luminosidad (Givnish 1988, Pearcy 1999, Lusk 2004).

Entre todas estas respuestas, variaciones en la capacidad fotosintética como respuesta a diferencias abióticas así como cambios en la disponibilidad de luz, agua o nutrientes ha sido ampliamente estudiada (e.g., Sims \& Pearcy 1992, Guariguata \& Kattan 2002, Wise \& Abrahamson 2005). Sin embargo, pese a la creciente fragmentación de los hábitats naturales, particularmente de bosques (Bustamante \& Grez 1995), de un total de 4.185 trabajos publicados sobre fotosíntesis entre los años 2000 y 2005, ninguno aborda las respuestas foliares en ambientes fragmentados (base de datos EBSCO Host e ISI Web of Science).

El bosque maulino costero es uno de los bosques más escasos y amenazados de Chile (Dinerstein et al. 1995, Echeverría et al. 2006). Estos fragmentos de bosque poseen aproximadamente $5 \%$ menos humedad de suelo, mayores temperaturas máximas diarias del aire y mayor radiación fotosintéticamente activa que el bosque continuo (Henríquez 2002, J.A. Simonetti resultados no publicados).

Entre las especies asociadas al bosque maulino y sus remanentes se encuentra Aristotelia chilensis, especie siempreverde reconocida por su plasticidad fisiológica (Lusk 2002), la que se expresa en una distribución amplia desde la provincia de Limarí (IV Región) hasta la de Aysén (XI Región), tanto en el valle central como en los faldeos cordilleranos, desde cerca del nivel del mar hasta los $2.500 \mathrm{~m}$ de altitud (Rodríguez et al. 1983). A baja luminosidad, A. chilensis presenta mayor área foliar específica que en situaciones de alta luminosidad (Lusk 2002, Lusk \& del Pozo 2002), respuesta foliar que le permitiría crecer en áreas abiertas y bajo el dosel de bosques (Damascos \& Prado 2001). Sin embargo, se desconoce otro tipo de respuestas morfológicas y sus consecuencias a nivel fisiológico en un ambiente donde se conjugan la sequedad y la luminosidad, como ocurre en un bosque fragmentado. 
En este contexto, el objetivo de este trabajo es estudiar las respuestas morfoquímicas a la fragmentación del bosque maulino y su repercusión a nivel fisiológico en hojas de Aristotelia chilensis. Específicamente, esperamos que las hojas de A. chilensis en fragmentos de bosque exhiban: (a) menor área foliar, menor área foliar específica, menor densidad de estomas, menor área estomática y menor concentración de nitrógeno con respecto a las hojas del bosque continuo y (b) mayor grosor de hoja y de la epidermis, mayor cantidad de tejido fotosintético (parénquima en empalizada y esponjoso), y mayor relación $\mathrm{C} / \mathrm{N}$ con respecto a las hojas del bosque continuo. Todas estas respuestas modificarían variables fisiológicas, por lo que hojas de fragmentos debieran ser capaces de mantener la capacidad fotosintética. Por ello, se esperaría además, que las hojas de fragmentos exhiban mayor eficiencia en el uso del agua, menor conductancia estomática y menor transpiración que en el bosque continuo.

\section{MATERIALES Y MÉTODOS}

\section{Sitio de estudio}

El bosque maulino costero se ubica en la costa de la Región del Maule (entre los $35^{\circ} 55^{\prime} \mathrm{S}$ y $37^{\circ} 20^{\prime}$ S) desde los 100 hasta $900 \mathrm{~m}$ de altitud. Este bosque alberga especies endémicas que se encuentran en peligro de extinción, tales como Gomortega keule (Molina) Baillon y Pitavia punctata (Molina). Las especies dominantes son Nothofagus glauca (Philippi) Krasser, Nothofagus obliqua (Mirbel) Oersted y Nothofagus alessandrii (Espinosa) asociados a Persea lingue (Nees), Gevuina avellana (Molina), Lithraea caustica (Molina) Hooker et Arnott y A. chilensis (San Martín \& Donoso 1996).

El estudio se realizó dentro del Sitio Prioritario Tregualemu, donde se consideró como área de bosque continuo a la Reserva Nacional Los Queules, un área protegida de 145 ha de bosque nativo inmersas en un área mayor de bosque continuo de 600 ha en total (Bustamante et al. 2005). En la periferia de la Reserva se encuentran pequeños parches de bosque maulino rodeado por plantaciones de Pinus radiata (David Don). La composición arbórea del bosque continuo y fragmentos es similar, siendo Aextoxicon punctatum Ruiz et Pavón, Cryptocarya alba (Molina) Looser, $G$. avellana, $N$. glauca y $P$. lingue las especies más frecuentes (Bustamante et al. 2005).

\section{Recolección de hojas}

Las muestras para morfología y química foliar así como las mediciones de intercambio de gases se realizaron sobre individuos reproductivos, de similar tamaño. De hecho, la distribución de tamaños, evaluada por DAP, no difiere entre el bosque continuo y los fragmentos (Lagos 2007). Todos los individuos estudiados estaban ubicados bajo el dosel de árboles emergentes, como $N$. glauca, en los centros geométricos de los fragmentos e interior del bosque continuo. Las muestras de hojas para morfología y contenido foliar fueron obtenidas de individuos creciendo en cuatro fragmentos diferentes, ubicados entre 0,5 y $4,5 \mathrm{~km}$ del bosque continuo. Los fragmentos se ubican entre 2,3 y $6,8 \mathrm{~km}$ entre sí (véase Donoso et al. 2003 para un mapa de ubicación).

\section{Morfología foliar}

El largo, ancho y área de las hojas se evaluó en hojas seleccionadas al azar en veinte individuos de A. chilensis en el bosque continuo y veinte individuos entre todos los fragmentos de bosque muestreados. En cada individuo se eligieron al azar aproximadamente 23 hojas sanas, totalizando una muestra de 925 hojas. El área foliar se estimó mediante un medidor de área foliar AM200 (ADC BioScientific ${ }^{\circledR}$ ), con un nivel de error en las mediciones de largo y ancho de $\pm 1 \mathrm{y} \pm 2 \%$ en las mediciones de área. Para evaluar el área foliar específica (SLA) se determinó el peso seco de las 925 hojas, luego de ser secadas en horno a $100{ }^{\circ} \mathrm{C}$ durante $48 \mathrm{~h}$. El área foliar específica de cada hoja se estimó como la razón entre el área de cada hoja y su peso seco $\left(\mathrm{cm}^{2} \mathrm{~g}^{-1}\right)$.

Para evaluar el tamaño de los estomas, así como la densidad y cantidad de estomas, se escogieron al azar cinco hojas por individuo (200 hojas en total) entre todas las hojas empleadas para las mediciones anteriores. La densidad de estomas se estimó a través de un microscopio óptico $(100 \mathrm{x})$, contando el número 
de estomas en un diámetro de $400 \mu \mathrm{m}$ (campo visual), en tres campos por muestra. La cantidad de estomas por hoja se estimó multiplicando la densidad de estomas por el área total de cada hoja. El tamaño de los estomas se determinó a partir de su área, la cual se midió a partir de fotos digitales, tomadas desde un microscopio óptico (400x), utilizando programa Micromeasure 3.3 en un total de 37 estomas en 15 individuos de fragmentos de bosque y 53 estomas en 20 individuos de bosque continuo.

Para evaluar la anatomía del tejido fotosintético, se escogió al azar una hoja por individuo, en cinco individuos de fragmentos y cinco individuos de bosque continuo. Las muestras fueron fijadas en formaldehídoalcohol-ácido acético por 48 horas, luego fueron colocadas por $2 \mathrm{~h}$ en una batería de alcoholes de grado ascendente (desde $50^{\circ}$ hasta $100^{\circ}$ ) y durante $8 \mathrm{~h}$ en terbutanol. Una vez deshidratadas, cada muestra fue incluida en Paraplast para realizar cortes histológicos con un micrótomo, los que fueron teñidos con safranina "fast-green" (Montenegro \& Riveros de la Puente 1977). El ancho de la epidermis superior e inferior, del parénquima en empalizada, la cantidad de capas de parénquima esponjoso y el porcentaje de espacio intercelular en esta zona, se estimó a través de observaciones con microscopia óptica $(300 \mathrm{X})$.

\section{Contenido foliar de nitrógeno y carbono}

Para determinar la concentración de nitrógeno y de carbono foliar, se colectaron al azar cinco hojas por individuo, en veinte individuos del bosque continuo y veinte individuos de los fragmentos. El porcentaje de carbono orgánico por peso seco se estimó a través de combustión húmeda y determinación colorimétrica al cromato reducido según Sadzawka et al. (2004). El porcentaje de nitrógeno en las hojas se estimó con el método de semimicro Kjeldahl y nesslerización directa (Longeri et al. 1979).

\section{Intercambio de gases}

Para evaluar la tasa fotosintética instantánea (expresada en $\mu \mathrm{mol} \mathrm{CO}_{2} \mathrm{~m}^{-2} \mathrm{~s}^{-1}$ ), transpiración $\left(\mu \mathrm{mol} \mathrm{H}_{2} \mathrm{O} \mathrm{m}^{-2} \mathrm{~s}^{-1}\right)$, conductancia estomática $\left(\mathrm{mmol} \mathrm{m} \mathrm{m}^{-2} \mathrm{~s}^{-1}\right)$ y uso eficiente del agua ( $\mu \mathrm{mol}$ $\mathrm{CO}_{2}$ por $\mu \mathrm{mol} \mathrm{H}_{2} \mathrm{O}$ ), se utilizó un analizador de intercambio gaseoso infrarrojo (IRGA) CIRAS II (PP Systems Haverhill, MA). Estas mediciones se realizaron en una cámara hermética que permite capturar una parte de la hoja $\left(2,5 \mathrm{~cm}^{2}\right)$, área desde la cual se estimó el intercambio gaseoso. Durante octubre 2005 (primavera) se realizaron las mediciones utilizando una fuente de luz de tungsteno alimentada por una batería, lo que permitió trabajar con una PPFD (Photosynthetic Photon Flux Density) de $100 \mu$ mol m $\mathrm{m}^{-2} \mathrm{~s}^{-1}$. Se seleccionaron al azar nueve árboles dentro del bosque continuo y cuatro árboles de un fragmento, en cada uno se escogió una hoja sana (sin seña de herbivoría o infección) sobre la cual se estimó el intercambio gaseoso (Lusk 2002).

\section{RESULTADOS}

\section{Morfología foliar}

Las hojas de árboles ubicados en fragmentos son 1,2 veces más cortas, con 1,2 veces menor área foliar y 1,2 veces menor SLA que aquellas de bosque continuo (Tabla 1). En cambio, el peso y ancho de las hojas no presentan ninguna diferencia significativa entre ambos hábitats (Tabla 1). Ni el tamaño de los estomas ni la densidad de estomas (número de estomas por $\mathrm{mm}^{2}$ ) difiere significativamente entre fragmentos de bosque y bosque continuo (Fig. 1). La cantidad promedio de estomas por hoja es 1,1 veces menor en individuos de fragmentos que en individuos de bosque continuo debido al menor tamaño de las hojas de fragmentos (Tabla 1). El grosor de la epidermis superior en hojas de fragmentos es 1,4 veces la del bosque continuo, y el grosor de la epidermis inferior solo 1,1 veces. El número de capas de parénquima esponjoso es 1,6 veces mayor en los fragmentos, por lo que el porcentaje de espacio intercelular es 1,9 veces mayor en las hojas del bosque continuo que en los fragmentos. El grosor del parénquima en empalizada no exhibió diferencias estadísticas significativas entre ambos ambientes, y el grosor de las hojas en los fragmentos es 1,2 veces mayor que en el bosque continuo (Tabla 1, Fig. 2). 


\section{TABLA 1}

Morfología foliar de Aristotelia chilensis en un bosque maulino fragmentado. Los valores corresponden a promedios y su error estándar asociado. Se incluye los estadígrafos U de MannWhitney y sus valores de probabilidad $(\mathrm{P})$ asociados. El número muestral fue de 20 individuos por tratamiento

Foliar morphology of Aristotelia chilensis in a fragmented maulino forest. Figures are mean values and standard errors. Mann-Whitney U statistics and their associated probability (P) values are given. Sample size was of 20 individuals per treatment

\begin{tabular}{|c|c|c|c|c|}
\hline Morfología foliar & Fragmentos & Bosque continuo & Valor de $\mathrm{U}$ & Valor de $\mathrm{P}$ \\
\hline Peso de hojas (g) & $0,11 \pm 0,01$ & $0,10 \pm 0,01$ & 192 & 0,83 \\
\hline Ancho (mm) & $34,02 \pm 0,9$ & $36,29 \pm 1,08$ & 141 & 0,11 \\
\hline Largo (mm) & $72,38 \pm 2,5$ & $90,14 \pm 1,67$ & 357 & $<0,01$ \\
\hline Área foliar $\left(\mathrm{cm}^{2}\right)$ & $16,19 \pm 0,85$ & $19,09 \pm 0,89$ & 118 & 0,03 \\
\hline Área foliar específica $\left(\mathrm{cm}^{2} \mathrm{~g}^{-1}\right)$ & $170,70 \pm 14,01$ & $202,31 \pm 8,80$ & 300 & 0,01 \\
\hline Densidad de estomas (estomas $\mathrm{mm}^{-2}$ ) & $183,85 \pm 5,26$ & $173,85 \pm 7,41$ & 159 & 0,26 \\
\hline Cantidad de estomas & $297.556,45 \pm 8516,19$ & $331.895,33 \pm 14150,29$ & 129 & 0,05 \\
\hline Área estomática $\left(\mu \mathrm{m}^{2}\right)$ & $0,0014 \pm 9,84 \mathrm{e}-5$ & $0,0016 \pm 11,92 \mathrm{e}-5$ & 801 & 0,14 \\
\hline Grosor de hojas $(\mu \mathrm{m})$ & $21,95 \pm 1,36$ & $18,23 \pm 0,22$ & 21 & 0,08 \\
\hline \multicolumn{5}{|l|}{ Grosor de cutícula $(\mu \mathrm{m})$} \\
\hline Epidermis adaxial & $3,41 \pm 0,18$ & $2,39 \pm 0,48$ & 23 & 0,03 \\
\hline Epidermis abaxial & $1,74 \pm 0,06$ & $1,51 \pm 0,02$ & 25 & 0,01 \\
\hline Parénquima en empalizada $(\mu \mathrm{m})$ & $6,07 \pm 0,26$ & $5,52 \pm 0,16$ & 20 & 0,12 \\
\hline \multicolumn{5}{|l|}{ Parénquima esponjoso } \\
\hline Número de capas & $4,43 \pm 0,30$ & $2,82 \pm 0,07$ & 25 & 0,01 \\
\hline Porcentaje vacío en la zona & $36 \% \pm 3,97$ & $68 \% \pm 1,37$ & 25 & 0,01 \\
\hline
\end{tabular}
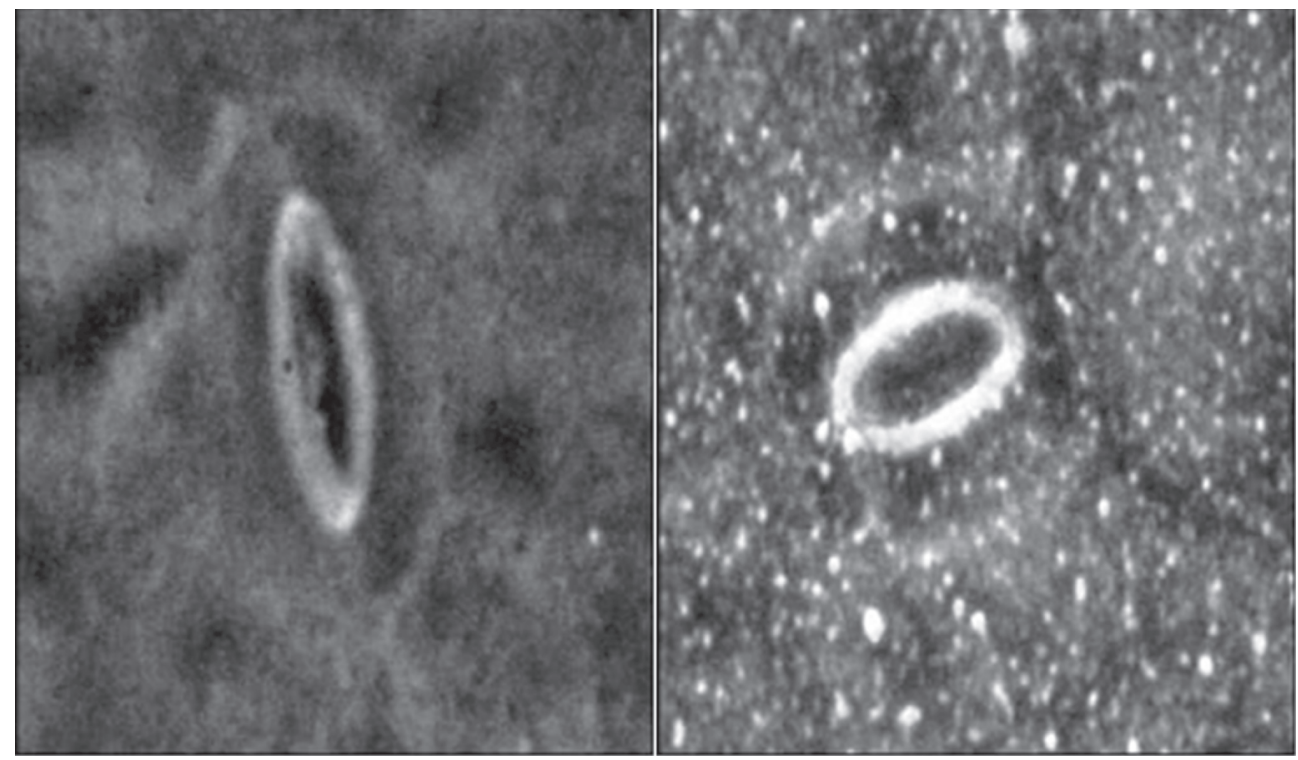

Fig. 1: Estomas de Aristotelia chilensis observados en microscopio óptico (400X). Se observa diferencia de largo en células guardianas, pero no del área de intercambio gaseoso. Estoma de hoja de fragmento de bosque (izquierda) y estoma de hoja de bosque continuo (derecha).

Stomata of Aristotelia chilensis observed in optical microscope (400X). Difference of length is observed in guardians cells, but not in the gaseous exchange area. Stomata of forest fragments (left) and stomata of continuous forest (right). 


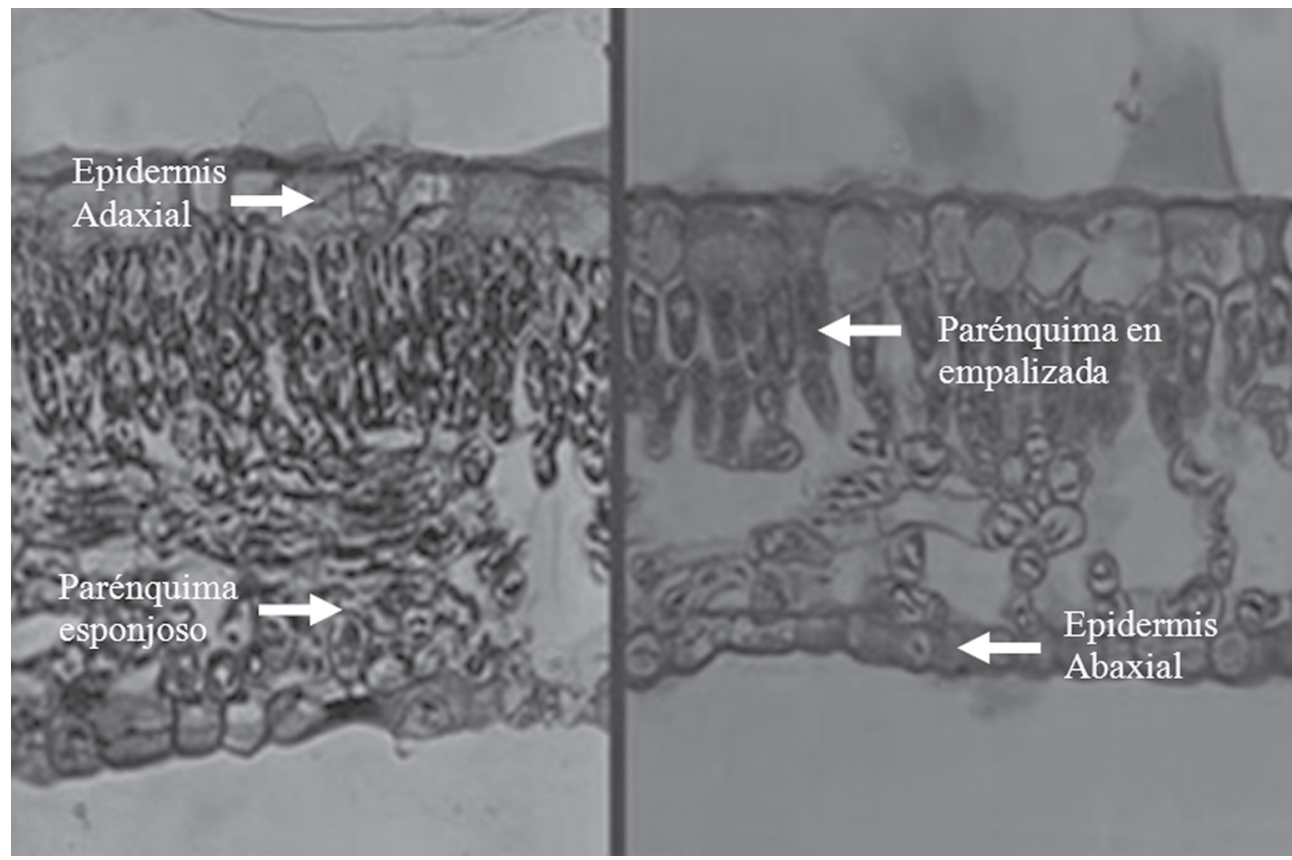

Fig. 2: Corte transversal de hoja de Aristotelia chilensis (300X) de fragmento de bosque (izquierda) y de bosque continuo (derecha). Se observa el mayor grosor de ambas epidermis y la mayor cantidad de parénquima esponjoso en la hoja de fragmento.

Transverse cut of Aristotelia chilensis leaves (300X) from fragments (left) and from continuous forest (right). Leaves from fragments exhibits larger spongy thickness of both epidermis and the larger quantity of parenchyma on fragment leaf.

\section{Contenido foliar de nitrógeno y carbono}

El porcentaje de nitrógeno por peso seco es 1,2 veces menor en las hojas de los fragmentos que en hojas del bosque continuo. Sin embargo, el porcentaje de carbono no difiere entre las hojas de ambos ambientes. Por lo tanto, la relación $\mathrm{C} / \mathrm{N}$ es 1,4 veces mayor en los fragmentos (Tabla 2).

\section{Intercambio de gases}

La tasa fotosintética neta y el uso eficiente del agua entre hojas de fragmentos y bosque continuo no difieren. La tasa de transpiración es 1,4 veces menor en hojas de fragmentos que en hojas del bosque continuo. La conductancia estomática no presenta diferencias significativas entre hojas de fragmentos y hojas de bosque continuo (Tabla 3 ).

TABLA 2

Contenido foliar de nitrógeno y carbono en Aristotelia chilensis en un bosque maulino fragmentado. Los valores corresponden a promedios y su error estándar asociado. Se incluye los estadígrafos U de Mann-Whitney y sus valores de probabilidad (P) asociados. El número muestral fue de 20 individuos por tratamiento

Nitrogen and carbon leaf contents in Aristotelia chilensis in a fragmented maulino forest. Figures are mean values and standard errors. Mann-Whitney U statistics and their associated probability (P) values are given. Sample size was 20 individuals per treatment

\begin{tabular}{lcccc}
\hline Química foliar & Fragmentos & Bosque continuo & Valor de U & Valor de P \\
\hline Nitrógeno (\% peso seco) & $0,90 \pm 0,10$ & $1,13 \pm 0,06$ & 66 & $<0,01$ \\
Carbono (\% peso seco) & $46,05 \pm 0,61$ & $44,29 \pm 0,74$ & 142 & 0,11 \\
Relación C/N & $56,95 \pm 3,63$ & $40,72 \pm 1,62$ & 55 & $<0,01$ \\
\hline
\end{tabular}


TABLA 3

Intercambio de gases en Aristotelia chilensis en un bosque maulino fragmentado. Los valores corresponden a promedios y su error estándar asociado. Se incluye los estadígrafos U de MannWhitney y sus valores de probabilidad $(\mathrm{P})$ asociados. El número muestral fue de cuatro individuos en fragmentos y nueve en bosque continuo

Gas exchange in Aristotelia chilensis in a forest fragmented maulino. Figures are mean values and standard errors. MannWhitney U statistics and their associated probability $(\mathrm{P})$ values are given. Sample size was four individuals in fragments and nine in continuous forest

\begin{tabular}{|c|c|c|c|c|}
\hline Intercambio de gases & Fragmentos & Bosque continuo & Valor de U & Valor de $\mathrm{P}$ \\
\hline Fotosíntesis neta $\left(\mu \mathrm{mol} \mathrm{CO} \mathrm{CO}^{-2} \mathrm{~s}^{-1}\right)$ & $5,38 \pm 0,72$ & $4,59 \pm 0,67$ & 22 & 0,53 \\
\hline Transpiración ( $\left.\mu \mathrm{mol} \mathrm{H}_{2} \mathrm{O} \mathrm{m}^{-2} \mathrm{~s}^{-1}\right)$ & $0,43 \pm 0,06$ & $0,61 \pm 0,05$ & 30 & 0,06 \\
\hline \multicolumn{5}{|l|}{ Eficiencia en el uso del agua } \\
\hline$\left(\mu \mathrm{mol} \mathrm{CO} \mathrm{CO}_{2}\right.$ por $\left.\mu \mathrm{mol} \mathrm{H}_{2} \mathrm{O}\right)$ & $15,61 \pm 4,42$ & $8,14 \pm 1,34$ & 24 & 0,35 \\
\hline Conductancia estomática $\left(\mathrm{mmol} \mathrm{m} \mathrm{m}^{-2} \mathrm{~s}^{-1}\right)$ & $26,50 \pm 3,95$ & $39,67 \pm 3,59$ & 28 & 0,12 \\
\hline
\end{tabular}

\section{DISCUSIÓN}

La fragmentación del bosque maulino provoca cambios ambientales en los fragmentos de bosque en comparación con el bosque continuo. Estas diferencias podrían implicar la sobrevivencia solo de aquellas especies capaces de responder a los cambios ambientales a través de modificaciones en su morfología y su fisiología, las que le permitirían maximizar la captura de energía, obteniendo mayor cantidad de recursos para reproducción (Givnish 1988). Por ejemplo, sobrevivir en un ambiente de baja humedad ambiental implica para las plantas un compromiso entre lograr una tasa fotosintética óptima con el mayor ahorro posible de agua, maximizando su uso eficiente (Roelfsema \& Hedrich 2005) y para aquellas plantas capaces de sobrevivir en ambientes de baja luminosidad implica incrementar la captura de energía por unidad de biomasa, por lo que maximizan su área foliar (Lusk 2004).

Aristotelia chilensis es una especie plástica. En fragmentos de bosque, A. chilensis disminuye la pérdida de agua reduciendo su superficie foliar, engrosando sus hojas, ambas epidermis foliares y aumentando la cantidad de capas de parénquima esponjoso. Al poseer menor área foliar, se reduce la cantidad de estomas en comparación con las hojas del bosque continuo. Todos estos cambios se reflejan en la menor transpiración por área total de las hojas de fragmentos de bosque comparado con el bosque continuo. En cambio, A. chilensis en el bosque continuo produce hojas más delgadas pero de mayor área que aquellas de fragmentos, aumentando con ello el área disponible para captar la radiación solar incidente en un ambiente donde es baja. Además, las hojas de bosque continuo concentran una mayor cantidad de nitrógeno que hojas de fragmento, lo que sugiere la presencia de una mayor cantidad de enzimas involucradas en la fotosíntesis (e.g., RUBISCO), aumentando así su capacidad para captar y utilizar en forma eficiente la baja radiación de estos ambientes (Walters \& Reich 1996).

Todas estas respuestas contrastantes entre un tipo de ambiente y el otro coinciden con las esperadas, indicando que los cambios observados en las hojas de A. chilensis le permiten maximizar la captura de energía tanto en fragmentos de bosque como en bosque continuo. Sin embargo, rasgos como densidad de estomas, tamaño de estomas, conductancia estomática y uso eficiente del agua no mostraron diferencias significativas entre hojas de fragmentos de bosque y hojas de bosque continuo. Estos rasgos se asocian principalmente a la regulación hídrica (Schulze \& Hall 1982), por lo que podría interpretarse como una falta de regulación estomática, debido a que los valores de estrés hídricos son moderados (Gulías et al. 2004), sugiriendo la predominancia de aquellas respuestas no estomáticas, como reducción de área foliar. Si bien la ausencia de diferencias significativas en los caracteres fisiológicos aquí evaluados podrían atribuirse al bajo número muestral, otros estudios con tamaños de muestra 
similares encuentran diferencias significativas en la capacidad fotosintética de algunas especies (e.g., Ngugi 2004 para especies del género Eucalyptus en ambientes con distinta disponibilidad de agua) lo que sugiere que la ausencia de diferencias en nuestro estudio sería un fenómeno biológico y no estadístico.

Aristotelia chilensis muestra una plasticidad fenotípica en rasgos asociados a la ganancia de carbono y economía del agua que le permite sobrevivir tanto en un hábitat con poca luz y mucha agua como el bosque continuo, y en uno con mucha luz y poca agua como los fragmentos de bosque. Esta plasticidad apoya la hipótesis que en un futuro próximo A. chilensis sería una de las especies dominantes de los fragmentos de bosque (Bustamante et al. 2005), ya que sus adaptaciones fisiológicas y morfológicas le permitirían sobrevivir y reproducirse en un medio ambiente de aceleradas transformaciones antropogénicas.

Respuestas similares en morfología foliar han sido documentadas en individuos de Colliguaja odorifera (Molina) creciendo en laderas de exposición norte -que reciben mayor cantidad de luz, son más secas y calientes- los cuales poseen características más xéricas que conespecíficos creciendo en laderas de exposición polar- que reciben menos luz, son más frías y húmedas (Montenegro \& Riveros de la Puente 1977). Además, C. odorifera presenta respuestas en la demografía foliar, exhibiendo períodos de emergencia de hojas más largos en condiciones de mayor disponibilidad de agua (Serey et al. 1987).

Finalmente, entre las 26 especies siempreverdes de los bosques de Chile que han sido estudiadas, al menos la mitad manifiesta un nivel intermedio de tolerancia a la sombra y un amplio rango de germinación (Figueroa \& Lusk 2001, Lusk 2002), lo cual -al igual que ocurre en A. chilensis- demuestra su plasticidad. Estas especies debieran ser analizadas en cuanto a sus respuestas morfológicas como fisiológicas en ambientes fragmentados, ya que estas respuestas podrían explicar, al menos parcialmente, la permanencia, invasión o extinción local desde los fragmentos del bosque nativo (Bustamante et al. 2005). Esta información es fundamental para diseñar e implementar reforestación y manejo de estrategias efectivas para el bosque nativo, incrementando además la comprensión de la ecología de los bosques.

\section{AGRADECIMIENTOS}

Agradecemos a Gloria Montenegro y Luis González (Pontificia Universidad Católica de Chile) su ayuda en los análisis morfológicos. Agradecemos también a Masisa S.A. y CONAF VII Región por permitirnos trabajar en sus terrenos. Este trabajo ha sido financiado por FONDECYT 1050745.

\section{LITERATURA CITADA}

BEERLING DJ \& WG CHALONER (1993) Evolutionary responses of stomatal density to global $\mathrm{CO}_{2}$ change. Biological Journal of the Linnean Society 48: 343 353.

BUCKLEY TN (2005) The control of stomata by water balance. New Phytologist 168: 275-292.

BUSTAMANTE RO \& AA GREZ (1995) Consecuencias ecológicas de la fragmentación de los bosques nativos. Ambiente y Desarrollo (Chile) 11: 58-63.

BUSTAMANTE RO, JA SIMONETTI, AA GREZ \& J SAN MARTÍN (2005) Fragmentación y dinámica de regeneración del bosque maulino: diagnóstico actual y perspectivas futuras. En: Smith-Ramírez C, JJ Armesto \& C Valdovinos (eds) Historia, biodiversidad y ecología de los bosques costeros de Chile: 555-564. Editorial Universitaria, Santiago, Chile.

CHAZDON RL \& S KAUFMANN (1993) Plasticity of leaf anatomy of two rain forest shrubs in relation to photosynthetic light acclimation. Functional Ecology 7: 385-394.

DAMASCOS MA \& CHBA PRADO (2001) Fenología foliar y sus caracteres asociados en la especie invierno-verde Aristotelia chilensis (Mol.) Stuntz (Elaeocarpaceae). Revista Chilena de Historia Natural 74: 805-815.

DAVIES WJ \& DJG COWING (1999) Plant responses to small perturbations in soil water status. En: Press MC, Scholes JD \& MG Barker (eds) Physiological plant ecology: 67-90. Blackwell Science, Oxford, United Kingdom.

DINERSTEIN E, DM OLSON, DJ GRAHAM, AL WEBSTER, SA PRIMM, MP BOOKBINDER \& G LEDEC (1995) A conservation assessment of the terrestrial ecoregions of latin America and the Caribbean. WWF \& The World Bank, Washington, District of Columbia, USA. 129 pp.

DONOSO DS, AA GREZ \& JA SIMONETTI (2003) Effects of forest fragmentation on the granivory of differently sized seeds. Biological Conservation 115: 63-70.

ECHEVERRÍA C, D COOMES, J SALAS, JM REYBENAYAS, A LARA \& A NEWTON (2006) Rapid deforestation and fragmentation of Chilean temperate forests. Biological Conservation 130: 481-494

FIGUEROA JA \& CH LUSK (2001) Germination requirements and seedling shade tolerance are not correlated in a Chilean temperate rain forest. New Phytologist 152: 483-489.

GIVNISH TJ (1988) Adaptation to sun vs shade: a wholeplant perspective. Australian Journal of Plant Physiology 15: 63-92. 
GUARIGUATA MR \& GH KATTAN (eds) (2002) Ecología y conservación de bosques neotropicales. Ediciones LUR, Cartago, Costa Rica. 619 pp.

GULÍAS J, J GALMÉS, J CIFRE, H MEDRANO \& J FLEXAS (2004) Ecofisiología de la fotosíntesis y la eficiencia en el uso del agua en la vegetación de la cuenca mediterránea. En: Cabrera HM (ed) Fisiología ecológica en plantas. Mecanismos y respuestas a estrés en los ecosistemas: 157-171. Ediciones Universitarias de Valparaíso, Valparaíso, Chile.

HARPER JL (1980) Plant demography and ecological theory. Oikos 35: 244-253.

HENRÍQUEZ CA (2002) El dilema de Lapageria rosea en bosques fragmentados ¿cantidad o calidad de la progenie? Tesis doctoral, Facultad de Ciencias, Universidad de Chile, Santiago, Chile. 133 pp.

KAPOS V, E WANDELLI, JL CAMARGO \& G GANADE (1997) Edge-related changes in environment and plant responses due to forest fragmentation in central Amazonia. En: Laurance WF \& Jr RO Bierregaard (eds) Tropical forest remnants. Ecology, management and conservation of fragmented communities: 33-44. University of Chicago, Chicago, Illinois, USA.

LAGOS N (2007) Patrón de distribución espacial de especies arbóreas en un bosque maulino fragmentado. Tesis Ingeniería en Recursos Naturales Renovables, Facultad de Ciencias Agronómicas, Universidad de Chile, Santiago, Chile.

LEAL DB \& SC THOMAS (2003) Vertical gradients and tree-to-tree variation in shoot morphology and foliar nitrogen in an old-growth Pinus strobes stand. Canadian Journal of Forestry Research 33: 1304-1314.

LERDAU M (2002) Benefits of the carbon-nutrient balance hypothesis. Oikos 98: 534-536.

LONGERI L, J ETCHEVERS \& J VENEGAS (1979) Metodología de perfusión para estudios de nitrificación en suelos. Ciencia e Investigación Agraria 6: 295-299.

LUSK CH (2002) Leaf area accumulation helps juvenile evergreen trees tolerate shade in a temperate rainforest. Oecologia 132: 188-196.

LUSK CH (2004) Adaptación a la sombra en especies arbóreas siempreverdes. En: Cabrera HM (ed) Fisiología ecológica en plantas. Mecanismos y respuestas a estrés en los ecosistemas: 235-247. Ediciones Universitarias de Valparaíso, Valparaíso, Chile.

LUSK CH \& A DEL POZO (2002) Survival and growth of seedlings of 12 Chilean rainforest trees in two light environments: gas exchange and biomass distribution correlates. Austral Ecology 27: 173182.

MONTENEGRO G \& F RIVEROS DE LA PUENTE (1977) Comparison of differential environmental responses of Colliguaja odorifera. Flora 166: 125 135.

MURCIA C (1995) Edge effects in fragmented forests: implications for conservation. Trends in Ecology and Evolution 10: 58-62.

NGUGI MR, D DOLEY, MA HUNT, P RYAN \& P DART (2004) Physiological responses to water stress in Eucalyptus cloeziana and Eucalyptus argophloia seedlings. Trees 18: 381-389.

PEARCY RW (1999) Responses of plants to heterogeneous light environments. En: Pugnaire FI \& F Valladares (eds) Handbook of functional plant ecology: 269-314. Marcel Dekker, New York, USA.

RODRÍGUEZ RR, OS MATTHEI \& MM QUEZADA (1983) Flora arbórea de Chile. Editorial Universitaria de Concepción, Concepción, Chile. $408 \mathrm{pp}$.

ROELFSEMA MRG \& R HEDRICH (2005) In the light of stomatal opening: new insight into "the watergate". New Phytologist 167: 665-691.

SADZAWKA AR, MR CARRASCO, RZ GREZ \& MG MORA (2004) Métodos de análisis recomendados para los suelos chilenos. Comisión de Normalización y Acreditación. Sociedad Chilena de la Ciencia del Suelo, Santiago, Chile. 113 pp.

SAN MARTÍN J \& C DONOSO (1996) Estructura florística e impacto antrópico en el bosque Maulino de Chile. En: Armesto JJ, C Villagrán y MK Arroyo (eds) Ecología de los bosques nativos de Chile: 153-168. Editorial Universitaria, Santiago, Chile.

SEREY I, M RICCI, E MANCILLA \& H MIRANDA (1987) Dinámica de las generaciones de hojas en el follaje de Colliguaja odorifera Mol. Medio Ambiente (Chile) 8: 22-26.

SCHULZE ED \& AE HALL (1982) Stomatal responses, water loss and $\mathrm{CO} 2$ assimilation rates of plants in contrasting environments. En: Lange OL, PS Nobel, $\mathrm{CB}$ Osmond \& $\mathrm{H}$ Ziegler (eds) Encyclopedia of plant physiology 12B: 181-230. Springer-Verlag, Berlin, Germany.

SILVA H, E ACEVEDO \& P SILVA (2001) Anatomía del tejido fotosintético de diez taxa de Opuntia establecidos en el secano árido mediterráneo de Chile. Revista Chilena de Historia Natural 74: 341351 .

SIMS DA \& RW PEARCY (1992) Response of leaf anatomy and photosynthetic capacity in Alocasia macrorrhiza (Araceae) to a transfer from low to high light. American Journal of Botany 79: 449-455.

WALTERS MB \& PB REICH (1996) Are shade tolerance, survival, and growth linked? Low light and nitrogen effects on hardwood seedlings. Ecology 77: 841853.

WISE MJ \& WG ABRAHAMSON (2005) Beyond the compensatory continuum: environmental resource levels and plant tolerance of herbivory. Oikos 109: 417-428. 
